\title{
Deciphering the mutation spectrum in south Indian children with congenital anomalies of the kidney and urinary tract
}

\author{
Ambili Narikot ${ }^{1}$, Varsha Chhotusing Pardeshi ${ }^{1}$, A. M. Shubha ${ }^{2}$, Arpana lyengar ${ }^{3}$ and Anil Vasudevan ${ }^{1,3^{*}}$
}

\begin{abstract}
Background: Congenital anomalies of the kidney and urinary tract (CAKUT) cover a spectrum of structural malformations that result from aberrant morphogenesis of kidney and urinary tract. It is the most prevalent cause of kidney failure in children. Hence, it is important from a clinical perspective to unravel the molecular etiology of kidney and urinary tract malformations. Causal variants in genes that direct various stages of development of kidney and urinary tract in fetal life have been identified in 5-20\% of CAKUT patients from Western countries. Recent advances in next generation sequencing technology and decreasing cost offer the opportunity to characterize the genetic profile of CAKUT in Indian population and facilitate integration of genetic diagnostics in care of children with CAKUT.

Methods: Customized targeted panel sequencing was performed to identify pathogenic variants in 31 genes known to cause human CAKUT in 69 south Indian children with CAKUT. The NGS data was filtered using standardized pipeline and the variants were classified using ACMG criteria. Genotype and phenotype correlations were performed.

Results: The cohort consisted of children mostly with posterior urethral valve (PUV) (39.1\%), vesico-ureteric reflux (VUR) (33.3\%) and multi-cystic dysplastic kidney (MCDK) (7.2\%). No pathogenic or likely pathogenic variants were identified in the study. Most of our variants $(n=39,60 \%)$ were variants of unknown significance with $25.6 \%$ (10/39) of them were identified as potentially damaging but were novel variants.

Conclusions: The present study did not identify any disease-causing monogenic variants in the cohort. The absence of genetic cause may be due to limitations of panel-based testing and also due to higher proportion of children with abnormalities in lower urinary tract than hypodysplasia of kidneys. Clinical, larger targeted panel or whole exome sequencing may be a better method to characterize the genetic profile of Indians patients with CAKUT.
\end{abstract}

Keywords: CAKUT, Genetics, Next generation sequencing, Variants, Monogenic

\section{Introduction}

Congenital anomalies of the kidney and urinary tract (CAKUT) comprises of a spectrum of defects involving kidney (hypoplasia and dysplasia), ureters and bladder (vesico-ureteral reflux, duplex system) and urethra (posterior urethral valves) that results from perturbations in

\footnotetext{
${ }^{*}$ Correspondence: anil.vasudevan@sjri.res.in

${ }^{3}$ Department of Pediatric Nephrology, St. John's Medical College, Bengaluru 560034, India

Full list of author information is available at the end of the article
}

the development of the kidney and urinary tract during fetal life $[1,2]$. The malformations may affect the kidney and urinary tract unilaterally and bilaterally or may occur in syndromic forms with other organ defects [3]. CAKUT occurs in about 5 per 1000 live births and contributes to $40-50 \%$ of end-stage kidney disease (ESKD) in children worldwide $[4,5]$.

Evidence from animal models, familial clustering and the occurrence of CAKUT in syndromic forms are indicative of genetic basis of the disease [6]. More than 
50 single gene defects have been identified in 10 to $12 \%$ of isolated CAKUT [7]. These are inherited in recessive or dominant form with incomplete penetrance and variable expressivity, resulting in genetic heterogeneity and complex inheritance of CAKUT. Detection of a causal variant in children with CAKUT is important for confirming the molecular cause, to facilitate early recognition of extra renal abnormalities and prompt treatment of clinically significant co-morbidities. In view of lack of genetic screening studies in Indian population, information about genes and the frequency of variants in these genes in Indian children with CAKUT is unknown which is a major barrier to the routine use of genetic testing in the clinic.

Significant advances in next-generation sequencing based genetic testing have immensely enhanced knowledge of genetic basis of many diseases and improved patient care and genetic counseling. Apart from costeffectiveness, heterogeneity in CAKUT with multiple genes being implicated with diverse modes of inheritance along with significant phenotypic variability justifies the use of next-generation sequencing (NGS) as a tool for genetic screening [7]. In conditions associated with multiple single gene defects, gene panel-based NGS is a cost effective method for the identification of casual variants in a specific set of disease associated genes and also prevents the identification of incidental variants [8-10]. However, selection of genes for the NGS panel and diagnostic yield has not been evaluated in most of the ethnic backgrounds, including in Indian population.

The aim of the present study was to determine the utility of NGS based customized targeted gene panel for genetic diagnosis of CAKUT and to know the genetic spectrum in south Indian children with CAKUT. We screened common CAKUT phenotypes in children using customized NGS based gene panel.

\section{Materials and methods \\ Patients and clinical data}

In this prospective study, children diagnosed with CAKUT (age group: newborn - 18years) were enrolled for the study from pediatric nephrology and surgery units between Jan 2016 and Jan 2019. Both incident and prevalent cases with follow up for at least 6 months were considered for recruitment. Institutional Ethics Committee approved the study (IEC Ref No- 163/2016) and all participants were recruited after informed consent. The diagnosis of CAKUT was made by pediatric nephrologists or surgeon on the basis of the following: ultrasonography (USG) of kidney and urinary tract, DMSA (Dimercaptosuccinic acid) scan and Micturating Cysto-urethrogram (MCUG), to identify any abnormality of number, size, shape, or anatomic position of the kidneys or other parts of the urinary tract. It included participants who had at least one of the following: vesicoureteral reflux (VUR) with or without hypodysplasia, posterior urethral valve (PUV), uretero-pelvic junction obstruction (UPJO), vesico-ureteric junction obstruction (VUJO), multicystic dysplastic kidney (MCDK), isolated renal hypodysplasia which is characterized by small kidney size with altered echogenicity, unilateral renal agenesis, duplex collecting system, megaureter and horseshoe kidney $[6,11]$. Both, syndromic (defined as CAKUT with extra renal features like optic nerve coloboma, ear fistula, pinna deformation, hearing loss, cardiac, gastrointestinal, neurological or skeletal defects) and non-syndromic cases (defined as CAKUT without extra renal features) were included in the study. Patients with a known causative genetic or chromosomal abnormality, isolated neurogenic bladder, and those who have undergone nephrectomy for medical reasons such as Wilm's tumor, refractory hypertension were excluded from the study. Clinical data, laboratory and imaging details were documented. Glomerular filtration rate (GFR) was estimated using revised Schwartz equation. Hypertension, chronic kidney disease (CKD) and ESKD was defined as per standard criteria [12]. For children less than 2 years, age-specific normal ranges for GFR was used to diagnose CKD [13].

\section{Next generation sequencing}

Blood sample (EDTA $5 \mathrm{ml}$ ) collected from patients at the time of recruitment was further processed for extraction of DNA. Genomic DNA was extracted from leukocytes using QIAamp blood mini kit (Qiagen, Hilden, Germany) as per manufacturer's instructions. Quantity of extracted DNA was estimated using Qubit fluorometric assay (Thermofisher scientific, MA, USA).

Genes for the customized panel were selected through a literature search in various online public databases such as OMIM (Online Mendelian Inheritance in Man) database (http://omim.org/) and PUBMED (http://www. ncbi.nlm.nih.gov/pubmed/) (Dec 2015). The following keywords were used: CAKUT, urinary tract anomalies or abnormalities, multicystic kidney dysplasia, vesico-ureteral reflux, duplex collecting system, posterior urethral valve, uretero-pelvic junction obstruction and renal/kidney diseases, genetic testing and mutations in CAKUT. Thirty-one genes associated with CAKUT were finally selected after a review of published studies in different ethnicities. Customized gene panel consisting of 31 genes was designed using Ion-Ampliseq primer designer (Life Technologies), targeting the coding regions of the genes (Additional file 1.docx- Genes included in the customized CAKUT NGS panel). The panel consisted of 825 primers (3 primer pools) with amplicon size ranging from 125 to $375 \mathrm{bp}$, targeting the exonic regions $(221.38 \mathrm{~kb}, 449$ 
exons) with coverage of $98.99 \%$. The uncovered regions were mainly repeat rich region making primer designing difficult.

Sixty-nine patients (3 syndromic children) with complete clinical and follow up data and representing various CAKUT phenotypes were selected for sequencing. The barcoded libraries were prepared using $20 \mathrm{ng}$ DNA and HQ-Ion AmpliSeq Library Kit 2.0 (Thermo Fisher Scientific Inc. USA) as per the manufacturer's instructions. Library quantity and quality was determined using Qubit fluorometric assay and Agilent BioAnalyzer High-Sensitivity DNA kit (Agilent Technologies, CA, USA), respectively. The libraries were amplified using Ion-Ampliseq Hi-Fi PCR mix, partially digested and phosphorylated and barcoded using Unique-Ion express barcodes. The barcoded libraries were pooled and enriched using Ion PGM Template OT2 400 kit (Thermo Fisher Scientific Inc. USA) according to the manufacturer's protocol. Next generation sequencing was carried out using the Ion PGM Hi-Q Sequencing Kit (318 Chip, Thermo Fisher Scientific Inc. USA) on Ion Torrent Personal Genome Machine sequencer (Thermo Fisher Scientific Inc. USA) as per the manufacturer's protocol.

\section{NGS data analysis}

Analysis was carried out using Ion Torrent Suite ${ }^{\mathrm{TM}}$ Browser version 5.0 and Ion Reporter ${ }^{\text {TM }}$ version 5.0. Torrent Suite ${ }^{\mathrm{TM}}$ Browser was used to perform initial quality control including chip loading density, base calling, alignment (hg19/GRCh37), median read length and number of mapped reads, assembly, coverage analysis and variant calling. Variants were identified by Ion Reporter using in house developed and validated filtering criteria: variant coverage $>20 x$,variant type and effect (non-synonymous, frame-shift, nonsense), location (to detect splice site variants) and variants with a minor allele frequency (MAF) $<1 \%$ in public databases (Gnomad (https://gnomad. broadinstitute.org/), ExAc and 5000 Exome (http://evs. gs.washington.edu/EVS/) [14]. All frame-shift variants and variants affecting stop codons were retained irrespective of their MAF. Synonymous variants except those located $\pm 2 \mathrm{bp}$ off exon boundaries and intronic variants $>10$ bp from exon boundaries were removed. Variants with a minor allele frequency (MAF) higher than 0.01 in online databases ExAC and 1000 genome, were excluded. The filtered variants were manually inspected with a high-performance visualization tool - Integrative Genomics Viewer (IGV) ( [15]) to filter out variants with possible strand-bias and variants within homopolymeric region. The functional significance of the filtered variants were evaluated in-silico using online prediction software SIFT [16], Polyphen-2 ( [17]) and MutationTaster [18]. Variants of interest were classified as pathogenic, likely pathogenic, variants of uncertain significance (VUS), likely benign, or benign using American College of Medical Genetics and Genomics (ACMG) guidelines and Sherloc guidelines by Nykamp et.al, which was a refinement of ACMG guidelines [19, 20]. A scoring system developed by Karbassi et al. was used to determine the pathogenicity of VUS [21].

\section{Results}

\section{Patient characteristics}

A flow diagram of recruitment process for CAKUT is shown in Fig. 1. A total of 128 patients were screened and 110 children diagnosed with CAKUT were included in the study. Eighteen children with no follow up data or without a conclusive diagnosis were excluded. All 110 patients could not be included for sequencing due to cost constraints. Patients with complete clinical data and follow up data of at least 6 months were selected for sequencing. Also, proportionate weightage was given to each diagnosis in entire cohort (Table 1). The clinical characteristics of patients included in the whole cohort $(n=110)$ as well as those sequenced $(n=69)$ is given in Table 1. PUV and VUR were most common diagnosis observed. In the sequenced cohort, 27 subjects had abnormalities of kidneys and urinary tract suggestive of CAKUT in antenatal ultrasound. In the rest CAKUT was diagnosed after birth, with median age at diagnosis of 1 year (IQR- 0.75 - 3) (Additional file 2: Table S1.xlsx -Detailed clinical profile of cohort included in the study). The median age at recruitment was 6 years (IQR- 4.5-9). The most common diagnosis was VUR $(n=42)$ and PUV $(n=37)$ (Table 1). History of consanguinity was observed in $15(21.73 \%)$ patients. Three patients had extra renal manifestations which included anorectal malformations (ARM), recto vestibular fistula, congenital heart defect (CHD), atrial septal defect (ASD) and urogenital sinus. Of the 69 subjects sequenced, 9 patients (13.04\%) exhibited impaired renal function (defined as $C K D \geq$ stage 2with estimated glomerular filtration rate $\leq 60 \mathrm{~mL} /$ $\mathrm{min} / 1.73 \mathrm{~m} 2)$ at the recruitment, and 3 of them (4.34\%) progressed to CKD at the median age of 12 years (IQR6-12) (Table 1).

\section{Genetic analysis}

Five sequencing runs to screen 69 subjects generated $1.57 \mathrm{~GB}$ of Q20 data. The coverage was comparable between runs (Additional file 2: Table S2.xlsx -Ion PGM next-Generation Sequencing run summary). Combining the data derived from five runs, sequencing generated a mean of $0.19 \mathrm{M}$ reads per individual with mean read length of $229 \mathrm{bp}$. On an average, $99.2 \%$ of these reads mapped to the reference genome and $97 \%$ were on target. Mean coverage of $190 \times$ was achieved for the genes 


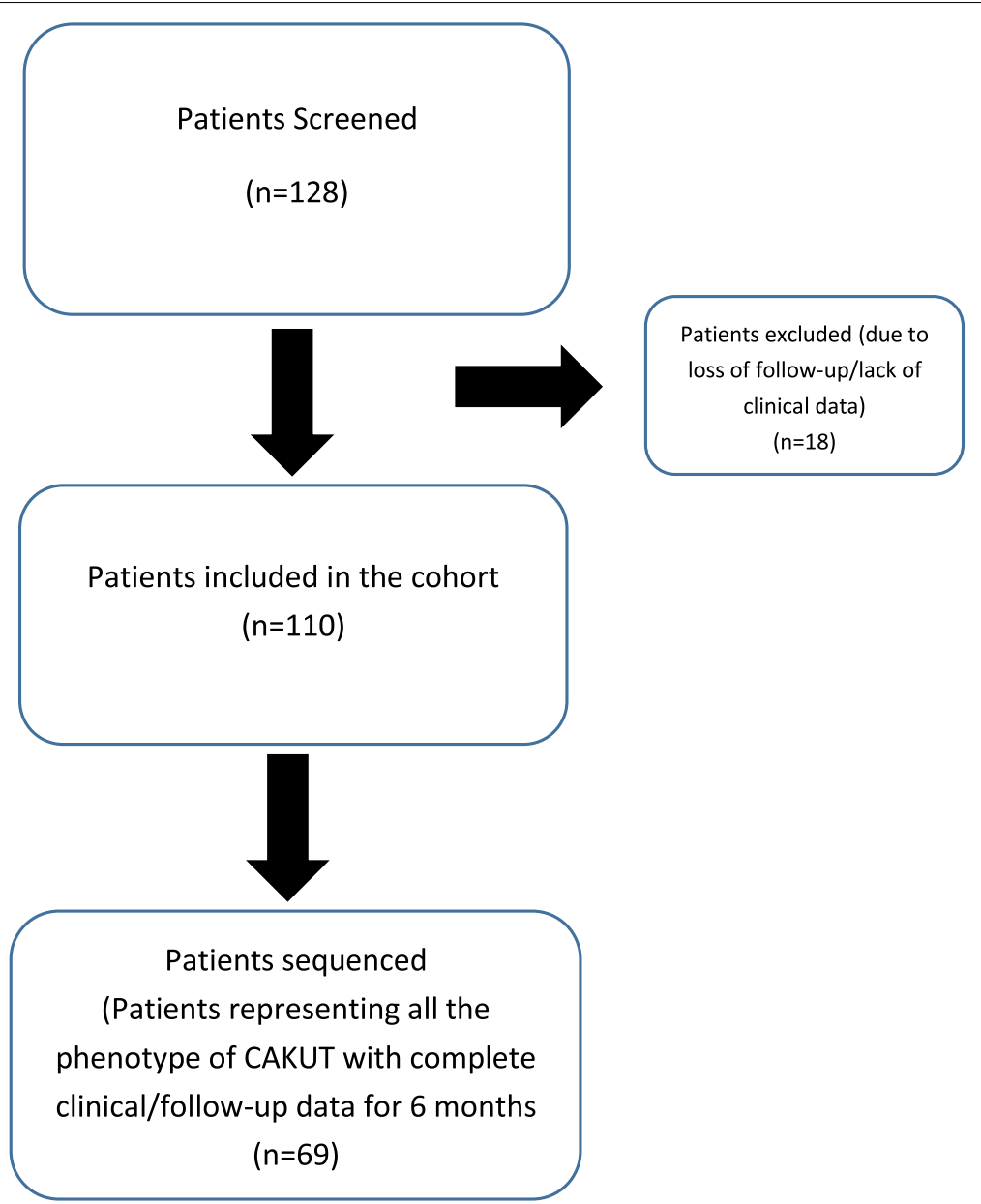

Fig. 1 Flowchart of the children with congenital anomalies of the kidney and urinary tract cohort development

across all samples, with 86,53 and $9 \%$ of the targets having minimum read depth of $20 \times, 100 \times$ and $500 \times$ respectively (Additional file 2: Table S3.xlsx -Summary of per sample NGS data output and quality in Indian Congenital Anomalies of Kidney and Urinary Tract cohort).

A total of 21,142 single-nucleotide variants (SNVs) and indels were obtained and these variants were annotated and filtered using Ion Reporter software 5.1 with parameters as given in Fig. 2. Following filtration, 367 variants were obtained which were reviewed manually, by examining quality scores and visually inspecting the data in Integrative Genome Viewer (IGV; Broad Institute). A total of 282 variants were removed subsequently due to strand bias, poor mapping in IGV, presence of variant in homopolymeric region and not well sequenced clean neighborhood. Following IGV, 85 variants were obtained, and from these 20 heterozygous variants identified in genes with recessive mode of inheritance were excluded. The remaining 65 variants were classified according to standard ACMG criteria; 39 variants of unknown significance (60\%) and 26 likely benign variants (40\%) were identified. Fourteen variants out of 65 variants (21.5\%) were novel. The lists of variants identified in each patient are listed in Additional file 2: Table S4.xlsx (List of per patient variant identified in the cohort).

\section{Genotype-phenotype correlation}

Majority $(60 \%)$ of identified variants were of unknown significance of which 14 variants were novel i.e. absent in population databases. Even though no pathogenic or likely pathogenic variants were identified in the study, 10 variants in 5 genes were identified as potentially damaging based on scoring criteria (CHD1L $(n=1)$, FRAS1 $(n=3)$, TNXB $(n=4)$, FREM2 $(\mathrm{n}=1), \operatorname{SIX5}(\mathrm{n}=1))$ (Additional file 2: Table S5.xlsx -Pathogenicity risk score calculation for Variant of unknown significance). The phenotypes observed in patients with these 7 variants were UPJO, duplex collecting system and renal agenesis for FRAS1 variants, VUR, L-Duplex ureter, PUV and horse-shoe kidney (syndromic) for $T N X B$ variants, VUR 
Table 1 Clinical characteristics of South Indian Children with congenital anomalies of the kidney and urinary tract

\begin{tabular}{|c|c|c|c|}
\hline Patient Characteristic & Study cohort $(n=110)$ & Sequenced Cohort $(n=69)$ & $p$ value* \\
\hline Age (yrs.) (median;IQR) & $5(5-9.2)$ & $6(4.5-9)$ & \\
\hline Gender (Male: Female) & $96: 14$ & 56: 13 & \\
\hline \multicolumn{4}{|l|}{ Renal Phenotypes: } \\
\hline 1. Vesico-Ureteric Reflux (VUR) (Bilateral; Unilateral) & $42(20: 22)(38.18 \%)$ & $23(14: 9)(33.33 \%)$ & 0.52 \\
\hline a. VUR with Hypodysplasia & $26(23.63 \%)$ & $10(14.49 \%)$ & \\
\hline b. VUR without Hypodysplasia & $16(14.54 \%)$ & $13(18.84 \%)$ & \\
\hline 2. Hypodysplastic kidney & $4(3.63 \%)$ & 0 & 0.30 \\
\hline 3. Posterior Urethral Valve (PUV) & $37(33.63 \%)$ & 27 (39.13\%) & 0.45 \\
\hline 4. Duplex Collecting System & $4(3.63 \%)$ & $3(4.34 \%)$ & 1.00 \\
\hline 5.Multi-Cystic Dysplastic Kidney (MCDK) & $7(6.36 \%)$ & $5(7.24 \%)$ & 1.00 \\
\hline 6. Ureteropelvic Junction Obstruction (UPJO) (Bilateral: Unilateral) & $5(1: 4,4.54 \%)$ & $5(1: 4,7.24 \%)$ & 0.51 \\
\hline 7. Vesicoureteral Junction Obstruction (VUJO) (Bilateral: Unilateral) & $2(0: 2,1.81 \%)$ & $2(0: 2,2.89 \%)$ & 0.64 \\
\hline 8. Renal Agenesis & $7(6.36 \%)$ & $3(4.34 \%)$ & 0.74 \\
\hline 9. Horseshoe Kidney & $1(0.9 \%)$ & $1(1.44 \%)$ & 1.00 \\
\hline 10. Ectopic kidney & $1(0.9 \%)$ & 0 & 1.00 \\
\hline Family history & $2(1.81 \%)$ & 0 & \\
\hline Consanguinity ${ }^{a}$ & $27(24.54 \%)$ & 15 (21.73\%) & \\
\hline Syndromic & $8(7.27 \%)$ & $3(4.34 \%)$ & \\
\hline No of subjects with CKD ( $\geq$ stage 2 ) @ recruitment & $21(19.09 \%)$ & $9(13.04 \%)$ & \\
\hline
\end{tabular}

${ }^{a}$ As reported by parents * chisquare test between diagnostic category in study cohort and sequenced cohort

for FREM2 variant, PUV for CHD1L gene and VUJO with Duplex system for variant in SIX5 gene. Patient CT-51, with PUV had variants in two genes, $[T N X B$ (p. Gln286fs), and CHD1L (p. Ser837fs)]. Another patient (CT-71) with similar TNXB (p. Gln286fs) gene variant had left duplex system with VUR in the lower moiety of left kidney. Two variants were identified in the same gene $(T N X B)$ in two patients with different malformations, but the type of variants reported were different. The child with PUV (CT-51) had a deletion (c.857delA) while an insertion (c.856_857insG) was identified at the same chromosome position (chr6:32064772) in child with duplex system (CT-71). Although three variants [TNXB (p. Gln286fs), CHD1L (p. Ser837fs) and TNXB (p. Gln286fs)] are loss-of-function variants and shown to be damaging using computational tools, they were classified as VUS because they are not constrained against Loss-of-function $(\mathrm{pli}=0)$. Three novel missense variants which were absent in Gnomad were identified in PAX2 (p.Ser305Leu in child with unilateral hypodysplastic kidney), SALL1 (p.Phe447Tyr in patient with bilateral VUR) and $R E T$ genes (p.Gly533Ser in child with unilateral vesico-ureteric junction obstruction).

\section{Discussion}

Identifying genetic etiology of CAKUT poses a unique challenge due to clinical variability and genetic heterogeneity. Targeted re-sequencing approach has shown great potential in simultaneous testing of large numbers of patients and multiple known disease causing genes in a various renal disorders [22-24]. Therefore, a customized gene panel of CAKUT was developed and tested for its utility in genetic diagnosis and to determine genetic profile of CAKUT in Indian children.

The present study $(n=69)$ included isolated and syndromic CAKUT cases representing the full spectrum of CAKUT phenotypes. Although, no pathogenic or likely pathogenic variants were identified, it is important to report VUS as these variants could be reclassified as pathogenic or likely pathogenic if additional patients with same phenotype and same variants are reported in future. After determining the pathogenic score of the VUS variants using Karbassiet.al. Scoring system, 10 VUS variants which are potentially damaging were identified in 5 genes (FRAS1, TNXB, FREM2, SIX5 and CHD1L). Interestingly two cases with different CAKUT phenotypes (case 51 with PUV and case 71 with L Duplex Ureter system) showed single gene variant $(T N X B)$ gene at the same position although the variants reported were different (c.857delA and c.856_857insG respectively). Previous Genome Wide Linkage Study (GWLS) and Whole Exome Sequencing (WES) study in a large kindred with VUR identified heterozygous pathogenic variants in TNXB as a cause of dominantly inherited VUR and joint hypermobility [25]. In the present study variant in TNXB gene was observed in duplex ureter system 


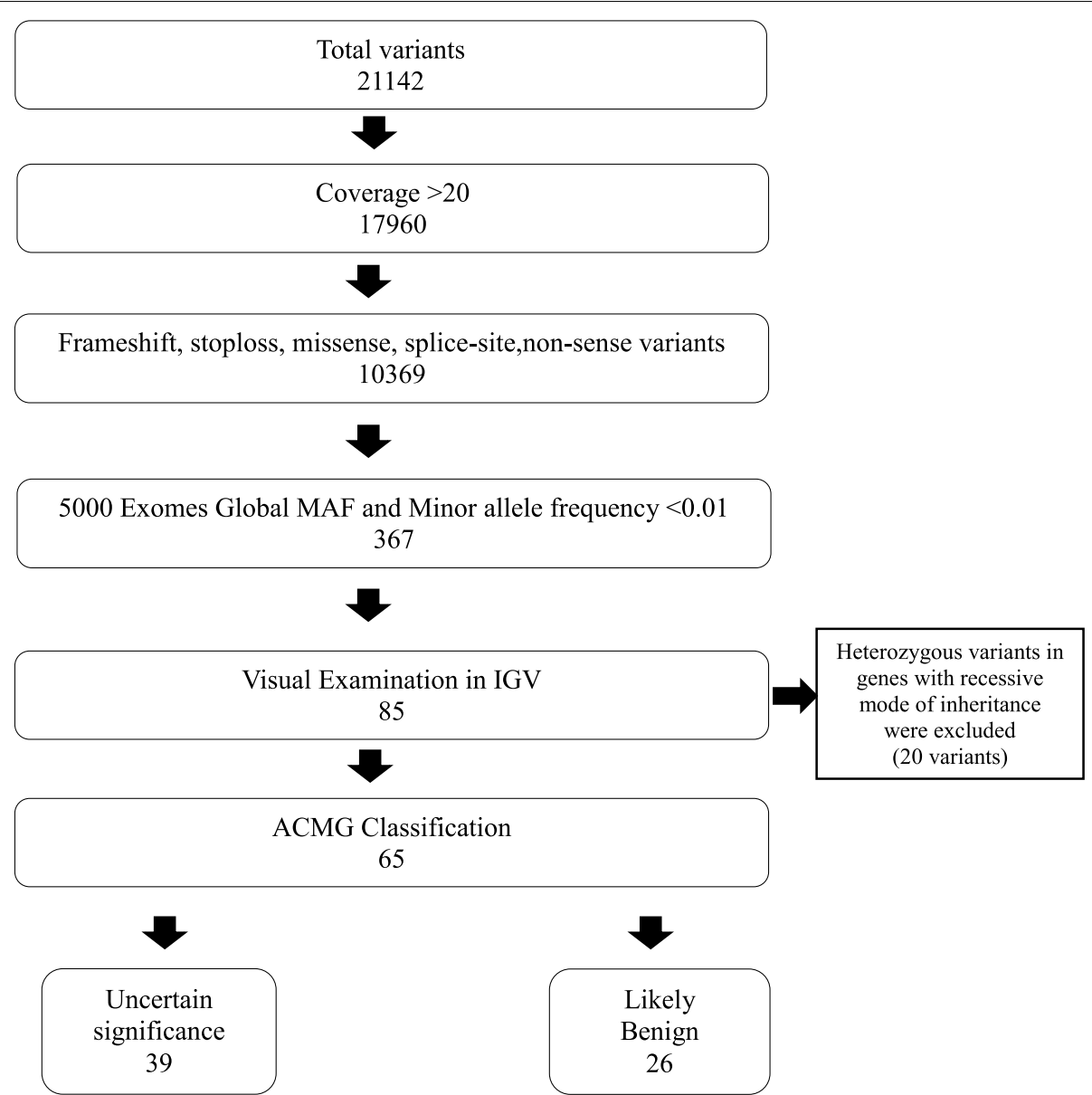

Fig. 2 Schematic visualization of the variant filtration workflow used in this study

phenotype with reflux in lower moiety. The patient with CHD1L variant showed a similar phenotype of PUV that has been reported previously in a study conducted by Hwang et al. Authors identified variant in CHD1L gene in five unrelated families $(0.76 \%)$ out of 650 families of diverse ethnic background. The phenotypes of individuals carrying the $C H D 1 L$ gene variant is very heterogenous: bilateral kidney malrotation, right renal dysplasia, horseshoe kidneys, right duplex collecting system, right MCDK, left UVJO and PUV [26]. Variants in two different genes may be due to the fact that kidney development is complex involving the interaction of distinct pathways. It is likely that defects in different genes involved in these pathways may result in similar phenotypes [11, 27-31]. These two variants identified in $C H D 1 L$ and TNXB met most of the criteria of pathogenicity but due to lack of data for the loss of function, these variants were further classified as variants of unknown significance. The findings in the current study supports the observations from previous studies that phenotypic classification alone may not be useful to predict the primary genetic defect in CAKUT [32]. For TNXB and FRAS1 gene variants, the observations were similar to that of previous studies; except for PUJO (FRAS1) and Horse-shoe kidney $(T N X B)$, which were observed only in our cohort [25, 26, 33-35]. Three novel missense variants were identified in PAX2, RET and SALL1 gene. Variant in PAX2 [p.Ser305Leu] was identified in a child (CT 14) with bilateral VUR and unilateral hypo dysplastic kidney. PAX2 belonging to the GDNF-RET signaling pathway plays a major role in morphogenesis and development of kidney and urinary tract [36]. PAX2 related disorder have a variable clinical presentation with renal and ophthalmological abnormalities (60-70\%), and sometimes with other abnormalities like hearing loss. On screening, child with the novel missense variant had no abnormality in the eye. In a Japanese cohort, of the seven patients with deleterious variants in $P A X 2$ gene who progressed to renal failure in childhood, six had truncating variants [37]. Like PAX2, SALL1 also belongs to GDNF-RET signaling pathway. A missense variant (p.Phe447Tyr) was identified in a child (CT 88) with bilateral VUR. Although heterozygous 
variants of SALL1 cause Townes-Brocks syndrome, comprising of facial dysmorphism, limb defects, kidney and urinary tract abnormalities and anorectal malformation, isolated kidney and urinary tract abnormalities have also been reported [38]. Targeted sequencing of 7 known CAKUT-causing dominant genes in 749 individuals identified novel missense variants in SALL1 in 6 patients, of which 4 had unilateral or bilateral VUR [26]. In the same study, deleterious variants in $R E T$ gene was identified in three families with different abnormalities of kidney and urinary tract suggesting phenotypic heterogeneity. In this study, RET variant (p.Gly533Ser) was identified in child (CT 73) with unilateral vesico-ureteric junction obstruction. Nevertheless, information about frequency of RET as one of the gene associated with CAKUT is conflicting $[39,40]$.

The diagnostic yield using targeted panel sequencing in CAKUT is variable with yield not impacted by number of genes included in the panel. Some studies reporting low diagnostic range $(2-15 \%)[1,26,41]$, while in few other studies, pathogenic variants were identified in $6-20 \%$ of patients with CAKUT by analyzing 5-30 genes [11, 34, $35,42-45]$.Study in a Dutch population also reported low diagnostic rate (3\%), where they sequenced 208 candidate genes in 453 subjects representing the full spectrum of CAKUT phenotypes [44]. However in another cohort, a higher molecular diagnosis rate (18\%) was reported in a panel sequencing study of 330 candidate genes in a cohort of 204 unrelated CAKUT patients [43]. In the current study many variants were classified as VUS, mainly as they were novel, lacking functional evidence, and also due to lack of evidence for segregation with the disease. In addition, a major barrier to variant calling is the absence of database specific to Indian population for allele frequencies. Larger multi-center study will also help in identifying common variants in children with CAKUT.

These findings indicate that the major limitation in variant classification is absence of functional data and genotype data from diverse ethnic background. With increasing NGS sequencing in clinical setting, it is likely that these VUS may be later classified as pathogenic or likely pathogenic. Previous studies reported $P A X 2$ and $H N F 1 B$ as the most frequently mutated genes in the European and American population with CAKUT. These studies predominantly consisted of children with renal hypodysplasia (RHD) [46-50]. This is in contrast to the present study, wherein children were non-syndromic (92\%) with lower urinary tract obstruction phenotypes -VUR (33.3\%) and PUV (39.1\%). HNF1B pathogenic variants are more frequently associated with hypo dysplasia and cystic kidneys and are rare in isolated lower urinary tract defects such as PUV and VUR, which were the most common diagnosis in the present study $[46,50]$. Hence it is not surprising that we did not find any deleterious variants in PAX2 or HNF1B.

The difference in the diagnostic yield in current study when compared to other studies could be attributed due to the approach using targeted panel as well as due to differences in cohort size, type of CAKUT that constituted the cohort and, the family history of patients, and also the genes included for screening. For example, high diagnostic yield was seen in cohort of severe CAKUT phenotypes, syndromic cases, patients from consanguineous families and familial cases [51, 52].This also addresses the need for familial testing as it helps in classification of variant into pathogenic or benign. But due to the unavailability of the samples, sequencing or testing of parents were not performed. It is possible that the deleterious variants in genes in targeted panel are rare in Indian population (less than $1 \%$ of observed cases with CAKUT) or larger cohorts screening is essential to confirm the observation. Besides monogenic cause, three large cohort studies identified that $4.5-16.6 \%$ of patients with CAKUT carry pathogenic CNV $[9,10,22,23,42,53-55]$ while targeted panel can identify single exon CNVs, multi exon CNVs are better identified using Whole Exome/genome sequencing panels. It is likely that CNVs in gene(s) or pathogenic variants in the noncoding regions not targeted by our customized panel could be responsible for the disease in the cohort. It is also known that epigenetic factors and non-genetic environmental factors also contribute to the occurrence of CAKUT [56]. This underlines that yet to be uncovered genetic complexity and environmental factors may be involved in the development of the disease. Hence, using other genetic testing approaches like microarray and clinical or whole exome sequencing may help decipher the genetic spectrum of CAKUT. WES studies reported diagnostic yield of $11-14 \%$ in CAKUT patients and identified novel gene variants $[1,56-60]$ which is higher than that obtained with targeted panel $[8,53-55]$. Exome sequencing have distinct advantages compared to targeted panel since it covers a larger number of genes as well identify other types of variants like CNVs but is more expensive compared to targeted sequencing. Targeted panels are phenotype-driven panels, featuring few hundred genes associated with kidney diseases. Its use in CAKUT may be limited due to significant phenotypic heterogeneity of CAKUT resulting in exclusion of genes associated with CAKUT of particular phenotype. It will be more useful to use exome sequencing based approach as many NGS-based targeted gene sets are built on an exome backbone. This allows the laboratory to use the same process for library preparation and sequencing leading to lesser cost. Such an approach not only 
facilitates targeted gene analysis but also in searching variants in remaining genes of clinical exome if targeted gene analysis fails to identify causal variants The low yield obtained using a targeted panel in this study and the need to frequently update the targeted panel for the newer discovered/novel CAKUT associated genes makes clinical exome sequencing a more favorable approach.

\section{Conclusion}

This is the first genetic analysis study conducted in Indian children with CAKUT using customized targeted panel sequencing. Most of our identified variants were of unknown significance, out of which 10 variants needs further exploration to determine pathogenicity. Using an exome sequencing approach and analyzing for $\mathrm{CNV}$ may help in identifying more children with CAKUT with a genetic cause and it can also help improve our understanding of genetic fingerprint in CAKUT.

\begin{abstract}
Abbreviations
CAKUT: Congenital Anomalies of Kidney And Urinary Tract; NGS: NextGenerationSequencing; USG: Ultrasonography; DMSA: Dimercapto succinic acid scan; MCUG: MicturatingCysto-Urethrogram; VUR: Vesico Ureteral Reflux; PUV: Posterior Urethral Valve; UPJO: Uretero Pelvic Junction Obstruction; VUJO: Vesico-Ureteric Junction Obstruction; MCDK: MultiCystic Dysplastic Kidney; RHD: Renal hypodysplasia; ARM: AnorectalMalformations; CHD: Congenital Heart Defect; ASD: Atrial Septal Defect; CKD: ChronicKidney Disease; IGV: Integrative Genomics Viewer; VUS: Variants of uncertain significance; ACMG: American College of Medical Genetics and Genomics; IQR: Inter Quartile Range; CNV: Copy Number Variation; WES: Whole Exome Sequencing; GWLS: Genome Wide Linkage Study.
\end{abstract}

\section{Supplementary Information}

The online version contains supplementary material available at https://doi. org/10.1186/s12882-021-02628-z.

\section{Additional file 1.}

Additional file 2 .

\section{Acknowledgements}

We thank all the patients who participated in the study.

\begin{abstract}
Authors' contributions
AN was involved in patient recruitment, performed the NGS run, analysed data and wrote the manuscript. VCP was involved in manuscript preparation. SAM and Al were involved in patient recruitment and manuscript review. AV originated the study, reviewed data analysis and co-wrote the manuscript. All authors read and approved the final manuscript.
\end{abstract}

\section{Funding}

This work was supported by a grant from Rajiv Gandhi University of Health sciences (RGU/ADV.RES/GRANTS/059/2016-17).

\section{Availability of data and materials}

The datasets generated and/or analysed during the current study are available in the GitHub repository [https://github.com/RenalGeneticsLab-SJRI/CAKUT].

\section{Declarations}

\section{Ethics approval and consent to participate}

The study has been approved by the institutional ethics committee (St.John's National Academy of Health Sciences Institutional Ethics committee, Bangalore, India, reference no 163/2016). Children < 18 years of age with CAKUT are enrolled in this study after written informed consent is obtained from parents or guardians.All methods were carried out in accordance with good clinical and laboratory practice requirements, and the applicable regulatory requirements.

\section{Consent for publication}

Written informed consent was obtained from the parents or quardians for publication. A copy of the written consent is available for review by the editor.

\section{Competing interests}

The authors declare that they have no competing interests.

\section{Author details}

${ }^{1}$ Divsion of Molecular Medicine, St. John's Research Institute, St. John's Medical College, Bengaluru, India. ${ }^{2}$ Department of Pediatric Surgery, St. John's Medical College, Bengaluru, India. ${ }^{3}$ Department of Pediatric Nephrology, St. John's Medical College, Bengaluru 560034, India.

Received: 17 April 2021 Accepted: 29 November 2021

Published online: 03 January 2022

\section{References}

1. van der Ven AT, Connaughton DM, Ityel H, Mann N, Nakayama M, Chen J, et al. Whole-exome sequencing identifies causative mutations in families with congenital anomalies of the kidney and urinary tract. J Am Soc Nephrol. 2018;29(9):2348-61.

2. Wühl E, van Stralen KJ, Verrina E, Bjerre A, Wanner C, Heaf JG, et al. Timing and outcome of renal replacement therapy in patients with congenital malformations of the kidney and urinary tract. Clin J Am Soc Nephrol [Internet]. 2013 Jan 7 [cited 2020 Jul 8];8(1):67-74. Available from: https:// pubmed.ncbi.n/m.nih.gov/23085722/

3. Song R, Yosypiv I V. Genetics of congenital anomalies of the kidney and urinary tract [Internet]. Vol. 26, Pediatric Nephrology. Pediatr Nephrol; 2011 [cited 2020 Jul 8]. p. 353-64. Available from: https://pubmed.ncbi. nlm.nih.gov/20798957/

4. Sanna-Cherchi S, Ravani P, Corbani V, Parodi S, Haupt R, Piaggio G, et al. Renal outcome in patients with congenital anomalies of the kidney and urinary tract. Kidney Int. 2009;76(5):528-33.

5. Harambat J, Van Stralen KJ, Kim JJ, Tizard EJ. Epidemiology of chronic kidney disease in children [Internet]. Vol. 27, Pediatric Nephrology. Springer Verlag; 2012 [cited 2020 Dec 16]. p. 363-73. Available from: https:// pubmed.ncbi.nlm.nih.gov/21713524/

6. Renkema KY, Winyard PJ, Skovorodkin IN, Levtchenko E, Hindryckx A, Jeanpierre C, et al. Novel perspectives for investigating congenital anomalies of the kidney and urinary tract (CAKUT). Nephrol Dial Transplant [Internet]. $2011 \mathrm{Dec}$ [cited $2020 \mathrm{Dec}$ 16];26(12):3843-51. Available from: https://pubmed.ncbi.nlm.nih.gov/22121240/

7. Capone VP, Morello W, Taroni F, Montini G. Genetics of congenital anomalies of the kidney and urinary tract: The current state of play [Internet] Vol. 18, International Journal of Molecular Sciences. MDPI AG; 2017 [cited 2020 Jul 8]. Available from: https://pubmed.ncbi.nlm.nih.gov/28398236/

8. Nicolaou N, Renkema KY, Bongers EMHF, Giles RH, Knoers NVAM. Genetic, environmental, and epigenetic factors involved in CAKUT. Vol. 11, Nature Reviews Nephrology. Nature Publishing Group; 2015. p. 720-731.

9. Hwang DY, Kohl S, Fan X, Vivante A, Chan S, Dworschak GC, et al. Mutations of the SLIT2-ROBO2 pathway genes SLIT2 and SRGAP1 confer risk for congenital anomalies of the kidney and urinary tract. Hum Genet [Internet]. 2015 Aug 23 [cited 2020 Jul 8];134(8):905-16. Available from: https://pubmed.ncbi.nlm.nih.gov/26026792/

10. Nigam A, Knoers NVAM, Renkema KY. Impact of next generation sequencing on our understanding of CAKUT. Vol. 91, Seminars in Cell and Developmental Biology. Elsevier Ltd; 2019. p. 104-110. 
11. Weber S, Moriniere V, Knüppel T, Charbit M, Dusek J, Ghiggeri GM, et al. Prevalence of mutations in renal developmental genes in children with renal hypodysplasia: Results of the ESCAPE study. J Am Soc Nephrol [Internet]. 2006 [cited 2020 Jul 8];17(10):2864-70. Available from: https:// pubmed.ncbi.n/m.nih.gov/16971658/

12. Flynn JT, Kaelber DC, Baker-Smith CM. Clinical Practice Guideline for Screening and Management of High Blood Pressure in Children and Adolescents [Internet]. Vol. 140, Pediatrics. 2017 [cited 2021 Mar 22]. Available from: www.aappublications.org/news

13. Heilbron DC, Holliday MA, Ai-Dahwi2 A, Kogan3 BA. Pediatric Nephrology Expressing glomerular filtration :rate in children. Vol. 5, Pediatr Nephrol. 1991.

14. Lek M, Karczewski KJ, Minikel E V., Samocha KE, Banks E, Fennell T, et al. Analysis of protein-coding genetic variation in 60,706 humans. Nature [Internet]. 2016 Aug 17 [cited 2020 Dec 16];536(7616):285-91. Available from: http://exac.broadinstitute.org

15. Thorvaldsdóttir H, Robinson JT, Mesirov JP. Integrative Genomics Viewer (IGV): High-performance genomics data visualization and exploration Brief Bioinform [Internet]. 2013 Mar [cited 2020 Dec 16];14(2):178-92. Available from: https://pubmed.ncbi.nlm.nih.gov/22517427/

16. Sim NL, Kumar P, Hu J, Henikoff S, Schneider G, Ng PC. SIFT web server: Predicting effects of amino acid substitutions on proteins. Nucleic Acids Res [Internet]. 2012 Jul [cited 2020 Dec 16];40(W1). Available from: https://pubmed.ncbi.nlm.nih.gov/22689647/

17. Adzhubei IA, Schmidt S, Peshkin L, Ramensky VE, Gerasimova A, Bork P, et al. A method and server for predicting damaging missense mutations [Internet]. Vol. 7, Nature Methods. NIH Public Access; 2010 [cited 2020 Dec 16]. p. 248-9. Available from: https://www.ncbi.nlm.nih.gov/pmc/ articles/PMC2855889/

18. Schwarz JM, Cooper DN, Schuelke M, Seelow D. Mutationtaster2: Mutation prediction for the deep-sequencing age [Internet]. Vol. 11, Nature Methods. Nature Publishing Group; 2014 [cited 2020 Dec 16]. p. 361-2. Available from: https://pubmed.ncbi.nlm.nih.gov/24681721/

19. Richards S, Aziz N, Bale S, Bick D, Das S, Gastier-Foster J, et al. Standards and guidelines for the interpretation of sequence variants: A joint consensus recommendation of the American College of Medical Genetics and Genomics and the Association for Molecular Pathology. Genet Med [Internet]. 2015 May 8 [cited 2020 Jul 8];17(5):405-24. Available from: https://pubmed.ncbi.nlm.nih.gov/25741868/

20. Nykamp K, Anderson M, Powers M, Garcia J, Herrera B, Ho YY, et al. Sherloc: A comprehensive refinement of the ACMG-AMP variant classification criteria. Genet Med [Internet]. 2017 Oct 1 [cited 2020 Jul 8];19(10):110517. Available from: https://pubmed.ncbi.nlm.nih.gov/28492532/

21. Karbassi I, Maston GA, Love A, Divincenzo C, Braastad CD, Elzinga CD, et al. A Standardized DNA Variant Scoring System for Pathogenicity Assessments in Mendelian Disorders. Hum Mutat [Internet]. 2016 Jan 1 [cited 2020 Jul 8];37(1):127-34. Available from: https://pubmed.ncbi.nlm. nih.gov/26467025/

22. M B el, PJ S. Next-generation Sequencing Technologies: Breaking the Sound Barrier of Human Genetics. Mutagenesis [Internet]. 2014 [cited 2020 Jul 8];29(5). Available from: https://pubmed.ncbi.nlm.nih.gov/25150023/

23. Leproust $E$. Target enrichment strategies for next generation sequencing. MLO Med Lab Obs. 2012;44(6):26-7.

24. Mamanova L, Coffey AJ, Scott CE, Kozarewa I, Turner EH, Kumar A, et al. Target-enrichment strategies for next-generation sequencing [Internet]. Vol. 7, Nature Methods. Nat Methods; 2010 [cited 2020 Jul 8]. p. 111-8. Available from: https://pubmed.ncbi.nlm.nih.gov/20111037/

25. Gbadegesin RA, Brophy PD, Adeyemo A, Hall G, Gupta IR, Hains D, et al. TNXB mutations can cause vesicoureteral reflux. J Am Soc Nephrol [Internet]. 2013 Jul 31 [cited 2020 Jul 8];24(8):1313-22. Available from: https:// pubmed.ncbi.nlm.nih.gov/23620400/

26. Hwang DY, Dworschak GC, Kohl S, Saisawat P, Vivante A, Hilger AC, et al. Mutations in 12 known dominant disease-causing genes clarify many congenital anomalies of the kidney and urinary tract. Kidney Int [Internet]. 2014 [cited 2020 Jul 8]:85(6):1429-33. Available from: https:// pubmed.ncbi.nlm.nih.gov/24429398/

27. Vainio S, Lin Y. Coordinating early kidney development: Lessons from gene targeting [Internet]. Vol. 3, Nature Reviews Genetics. Nat Rev Genet; 2002 [cited 2020 Jul 8]. p. 533-43. Available from: https://pubmed.ncbi. nlm.nih.gov/12094231/

28. Yu J, McMahon AP, Valerius MT. Recent genetic studies of mouse kidney development [Internet]. Vol. 14, Current Opinion in Genetics and
Development. Curr Opin Genet Dev; 2004 [cited 2020 Jul 8]. p. 550-7. Available from: https://pubmed.ncbi.nlm.nih.gov/15380247/

29. Miyazaki Y, Oshima K, Fogo A, Ichikawa I. Evidence that bone morphogenetic protein 4 has multiple biological functions during kidney and urinary tract development. Kidney Int [Internet]. 2003 Mar 1 [cited 2020 Jul 8];63(3):835-44. Available from: https://pubmed.ncbi.nlm.nih.gov/ 12631064/

30. Torres M, Gómez-Pardo E, Dressler GR, Gruss P. Pax-2 controls multiple steps of urogenital development. Development. 1995;121(12):4057-65.

31. Grieshammer U, Ma L, Plump AS, Wang F, Tessier-Lavigne M, Martin GR. SLIT2-Mediated ROBO2 signaling restricts kidney induction to a single site. Dev Cell [Internet]. 2004 May [cited 2020 Jul 8];6(5):709-17. Available from: https://pubmed.ncbi.nlm.nih.gov/15130495/

32. Sanna-Cherchi S, Caridi G, Weng PL, Scolari F, Perfumo F, Gharavi AG, et al. Genetic approaches to human renal agenesis/hypoplasia and dysplasia [Internet]. Vol. 22, Pediatric Nephrology. Springer Verlag; 2007 [cited 2020 Jul 8]. p. 1675-84. Available from: https://pubmed.ncbi.nlm.nih.gov/ $17437132 /$

33. Al-Hamed MH, Sayer JA, Alsahan N, Tulbah M, Kurdi W, Ambusaidi Q, et al. Novel loss of function variants in FRAS1 AND FREM2 underlie renal agenesis in consanguineous families. J Nephrol [Internet]. 2020 Jul 8 [cited 2020 Aug 6]; Available from: http://link.springer.com/10.1007/ s40620-020-00795-0

34. Kohl S, Hwang DY, Dworschak GC, Hilger AC, Saisawat P, Vivante A, et al. Mild recessive mutations in six Fraser syndrome-related genes cause isolated congenital anomalies of the kidney and urinary tract. J Am Soc Nephrol. 2014;25(9):1917-22.

35. Saisawat $P$, Tasic $V$, Vega-Warner $V$, Kehinde EO, Günther B, Airik R, et al. Identification of two novel CAKUT-causing genes by massively parallel exon resequencing of candidate genes in patients with unilateral renal agenesis. Kidney Int [Internet]. 2012 Jan 2 [cited 2020 Jul 8];81(2):196200. Available from: https://pubmed.ncbi.nlm.nih.gov/21900877/

36. F C, R S. GDNF/Ret signaling and the development of the kidney. Bioessays [Internet]. 2006 Feb [cited 2021 Aug 7];28(2):117-27. Available from: https://pubmed.ncbi.nlm.nih.gov/16435290/

37. Rossanti R, Morisada N, Nozu K, Kamei K, Horinouchi T, Yamamura T, et al. Clinical and genetic variability of PAX2-related disorder in the Japanese population. J Hum Genet 2020656 [Internet]. 2020 Mar 16 [cited 2021 Aug 7];65(6):541-9. Available from: https://www.nature.com/articles/ s10038-020-0741-y

38. Kohlhase J, Wischermann A, Reichenbach H, Froster U, Engel W. Mutations in the SALL1 putative transcription factor gene cause TownesBrocks syndrome. Nat Genet 1998181 [Internet]. 1998 [cited 2021 Aug 7];18(1):81-3. Available from: https://www.nature.com/articles/ng0198-81

39. $C J, G M, M P, V M, A P, M B$, et al. RET and GDNF mutations are rare in fetuses with renal agenesis or other severe kidney development defects. J Med Genet [Internet]. 2011 Jul [cited 2021 Aug 7];48(7):497-504. Available from: https://pubmed.ncbi.nlm.nih.gov/21490379/

40. AP P, M M I I , G M, C G, GM G, et al. Hirschsprung disease and congenital anomalies of the kidney and urinary tract (CAKUT): a novel syndromic association. Medicine (Baltimore) [Internet]. 2009 Mar [cited 2021 Aug 7];88(2):83-90. Available from: https://pubmed.ncbi.nlm.nih.gov/19282698/

41. Ishiwa S, Sato M, Morisada N, Nishi K, Kanamori T, Okutsu M, et al. Association between the clinical presentation of congenital anomalies of the kidney and urinary tract (CAKUT) and gene mutations: an analysis of 66 patients at a single institution. Pediatr Nephrol. 2019;34(8):1457-64.

42. Mu W, Lu HM, Chen J, Li S, Elliott AM. Sanger Confirmation Is Required to Achieve Optimal Sensitivity and Specificity in Next-Generation Sequencing Panel Testing. J Mol Diagnostics [Internet]. 2016 Nov 1 [cited 2020 Jul 8];18(6):923-32. Available from: https://pubmed.ncbi.nlm.nih.gov/27720 647/

43. Heidet L, Morinière $V$, Henry $C$, De Tomasi L, Reilly ML, Humbert $C$, et al. Targeted exome sequencing identifies PBX1 as involved in monogenic congenital anomalies of the kidney and urinary tract. J Am Soc Nephrol [Internet]. 2017 Oct 1 [cited 2020 Jul 8];28(10):2901-14. Available from: https://pubmed.ncbi.nlm.nih.gov/28566479/

44. Nicolaou N, Pulit SL, Nijman IJ, Monroe GR, Feitz WFJ, Schreuder MF, et al. Prioritization and burden analysis of rare variants in 208 candidate genes suggest they do not play a major role in CAKUT. Kidney Int [Internet]. 2016 Feb 1 [cited 2020 Jul 8];89(2):476-86. Available from: https://pubmed.ncbi.nlm.nih.gov/26489027/ 
45. McGregor L, Makela V, Darling SM, Vrontou S, Chalepakis G, Roberts C, et al. Fraser syndrome and mouse blebbed phenotype caused by mutations in FRAS1/Fras1 encoding a putative extracellular matrix protein. Nat Genet [Internet]. 2003 Jun 1 [cited 2020 Jul 8];34(2):203-8. Available from: https://pubmed.ncbi.nlm.nih.gov/12766769/

46. Heidet L, Decramer S, Pawtowski A, Morinière $V$, Bandin F, Knebelmann $B$, et al. Spectrum of HNF1B mutations in a large cohort of patients who harbor renal diseases. Clin J Am Soc Nephrol [Internet]. 2010 Jun 1 [cited $2020 \mathrm{Jul}$ 8];5(6):1079-90. Available from: https://pubmed.ncbi.nlm.nih. gov/20378641/

47. Nishimoto K, lijima K, Shirakawa T, Kitagawa K, Satomura K, Nakamura H, et al. PAX2 gene mutation in a family with isolated renal hypoplasia. J Am Soc Nephrol. 2001;12(8):1769-72.

48. Ulinski T, Lescure S, Beaufils S, Guigonis V, Decramer S, Morin D, et al. Renal phenotypes related to hepatocyte nuclear factor-1 $\beta$ (TCF2) mutations in a pediatric cohort. J Am Soc Nephrol [Internet]. 2006 Feb [cited 2020 Jul 8];17(2):497-503. Available from: https://pubmed.ncbi.nlm.nih. gov/16371430/

49. Madariaga L, Morinière $V$, Jeanpierre C, Bouvier R, Loget $P$, Martinovic $J$, et al. Severe prenatal renal anomalies associated with mutations in HNF1B or PAX2 genes. Clin J Am Soc Nephrol [Internet]. 2013 Jul [cited 2020 Jul 8];8(7):1179-87. Available from: https://pubmed.ncbi.nlm.nih. gov/23539225/

50. Thomas R, Sanna-Cherchi S, Warady BA, Furth SL, Kaskel FJ, Gharavi AG. HNF1B and PAX2 mutations are a common cause of renal hypodysplasia in the CKiD cohort. Pediatr Nephrol [Internet]. 2011 Jun [cited 2020 Jul 8];26(6):897-903. Available from: https://pubmed.ncbi.nlm.nih.gov/ 21380624/

51. Ahn YH, Lee C, Kim NKD, Park E, Kang HG, Ha I-S, et al. Targeted Exome Sequencing Provided Comprehensive Genetic Diagnosis of Congenital Anomalies of the Kidney and Urinary Tract. J Clin Med [Internet]. 2020 Mar 10 [cited 2020 Dec 17];9(3):751. Available from: https://pubmed.ncbi. nlm.nih.gov/32164334/

52. Westland R, Renkema KY, Knoers NVAM. Clinical integration of genome diagnostics for congenital anomalies of the kidney and urinary tract. Clin J Am Soc Nephrol [Internet]. 2020 Apr 20 [cited 2020 Dec 17];CJN.14661119. Available from: 10.2215/CJN.14661119.

53. Sanna-Cherchi S, Kiryluk K, Burgess KE, Bodria M, Sampson MG, Hadley D, et al. Copy-number disorders are a common cause of congenital kidney malformations. Am J Hum Genet. 2012;91(6):987-97.

54. Verbitsky M, Sanna-Cherchi S, Fasel DA, Levy B, Kiryluk K, Wuttke M, et al. Genomic imbalances in pediatric patients with chronic kidney disease. J Clin Invest. 2015;125(5):2171-8.

55. Westland R, Verbitsky M, Vukojevic K, Perry BJ, Fasel DA, Zwijnenburg PJG, et al. Copy number variation analysis identifies novel CAKUT candidate genes in children with a solitary functioning kidney. Kidney Int. 2015;88(6):1402-10

56. Ichikawa I, Kuwayama F, Pope JC, Stephens FD, Miyazaki Y. Paradigm shift from classic anatomic theories to contemporary cell biological views of CAKUT. Kidney Int [Internet]. 2002 [cited 2020 Jul 7];61(3):889-98. Available from: https://pubmed.ncbi.nlm.nih.gov/11849443/

57. Sanna-Cherchi S, Westland R, Ghiggeri GM, Gharavi AG. Genetic basis of human congenital anomalies of the kidney and urinary tract. Journal of Clinical Investigation. 2018.

58. Nakanishi K, Yoshikawa N. Genetic disorders of human congenital anomalies of the kidney and urinary tract (CAKUT) [Internet]. Vol. 45, Pediatrics International. Pediatr Int; 2003 [cited 2020 Jul 8]. p. 610-6. Available from: https://pubmed.ncbi.nlm.nih.gov/14521546/

59. Bekheirnia MR, Bekheirnia N, Bainbridge MN, Gu S, Coban Akdemir ZH, Gambin T, et al. Whole-exome sequencing in the molecular diagnosis of individuals with congenital anomalies of the kidney and urinary tract and identification of a new causative gene. Genet Med. 2017;19(4):412-20.

60. Lei T-Y, Fu F, Li R, Wang D, Wang R-Y, Jing X-Y, et al. Whole-exome sequencing for prenatal diagnosis of fetuses with congenital anomalies of the kidney and urinary tract. Nephrol Dial Transplant Off Publ Eur Dial Transpl Assoc - Eur Ren Assoc. 2017;32(10):1665-75.

\section{Publisher's Note}

Springer Nature remains neutral with regard to jurisdictional claims in published maps and institutional affiliations.
Ready to submit your research? Choose BMC and benefit from:

- fast, convenient online submission

- thorough peer review by experienced researchers in your field

- rapid publication on acceptance

- support for research data, including large and complex data types

- gold Open Access which fosters wider collaboration and increased citations

- maximum visibility for your research: over $100 \mathrm{M}$ website views per year

At BMC, research is always in progress.

Learn more biomedcentral.com/submissions 